\title{
Demodicose bovina no Estado da Paraíba ${ }^{1}$
}

\author{
João Luiz H. Faccini ${ }^{2}$, Ana Clara G. Santos ${ }^{3}$ e Gervásio H. Bechara ${ }^{4}$
}

\begin{abstract}
Faccini J.L.H., Santos A.C.G. \& Bechara G.H. 2004. [Bovine demodicosis in the state of Paraíba, northeastern Brazil.] Demodicose bovina no Estado da Paraíba. Pesquisa Veterinária Brasileira 24(3):149-153. Depto Parasitologia Animal, Instituto de Veterinária, Universidade Federal Rural do Rio de Janeiro, Seropédica, RJ 23890-000, Brazil. E-mail: faccini@ufrrj.br

Cases of bovine demodicosis caused by Demodex bovis were reported in a Sindhi herd from December 1989 to January 1992. Both localized and generalized forms were diagnosed. This is the first report of the generalized form in Brazil. In the first two years, demodicosis was diagnosed only in cattle $<2$ years old, whereas animals of all ages were positive in the last two years. Prevalence varied from $20.4 \%(11 / 54)$ to $53.1 \%(26 / 49)$ and $13.2 \%(12 / 91)$ to $14.8 \%(9 / 61)$ for cattle $<2$ years old and $>2$ years old, respectively. Clinical signs varied from a few small nodules to a thickened skin with soft large nodules in the localized and generalized forms, respectively. Main microscopic features of the nodules in the generalized form consisted of acanthosis with hyperqueratosis, chronic sebaceous adenitis, subcutaneous muscular necrosis, focal cellular degeneration of the epidermis basal layer and presence of large number of mites inside the lumen of dilated hair follicles. In addition, a chronic perifoliculitis was observed, characterized by lymphoplasmocytic infiltrate which also contained macrophages and neutrophils. It is suggested that poor nutrition and stress due to prolonged drought probably contributed to the increase of susceptibility of the herd to mite infestation.
\end{abstract}

INDEX TERMS: Demodex bovis, demodicosis, cattle, pathology, Brazil.

RESUMO.- Casos de demodicose bovina foram observados em um rebanho da raça Sindhi no período de dezembro 1989 a janeiro 1992. Ambas as formas, localizada e generalizada, foram diagnosticadas. Este é o primeiro relato da forma generalizada no Brasil. Nos dois primeiros anos, a demodicose foi diagnosticada somente em animais $<2$ anos, enquanto que nos dois últimos anos, animais de todas as idades estavam positivos. A prevalência variou de $20,4 \%(11 / 54)$ a $53,1 \%(26 / 49)$ e $13,2 \%(12 / 91)$ a $14,8 \%$ (9/61) nos animais com menos e mais de 2 anos de idade, respectivamente. Os sinais clínicos variaram de pequenos nódulos a espessamento da pele com nódulos grandes e moles, nas formas localizada e generalizada, respectivamente. Os principais achados histopatológicos dos nódulos cutâneos na forma generali-

\footnotetext{
${ }^{1}$ Recebido em 9 de setembro de 2003.

Aceito para publicação em 28 de maio de 2004

2 Depto Parasitologia Animal, Instituto de Veterinária, Universidade Federal Rural do Rio de Janeiro (UFRRJ), Seropédica, RJ 23890-000. Bolsista do CNPq. E-mail: faccini@ufrrj.br

${ }^{3}$ Depto Medicina Veterinária, CSTR, UFCG, Patos, PB 58700-000. (Aposentada)

${ }^{4}$ Depto Patologia Veterinária, FCAV, Unesp, Jaboticabal, SP 14870 000. Bolsista do CNPq.
}

zada foram: acantose com hiperqueratose, adenite sebácea crônica, necrose muscular subcutânea e degeneração focal de células da camada basal da epiderme, além da presença de grande número de ácaros de localização intraluminal nos terços médio e inferior de folículos pilosos dilatados. Observou-se ainda perifoliculite crônica, caracterizada por infiltrado celular predominantemente linfoplasmocitário contendo ainda macrófagos e neutrófilos, que envolvia bulbos pilosos. 0 estado nutricional precário dos animais e o estresse provocado pela longa estiagem, provavelmente, contribuíram para aumentar a suscetibilidade do rebanho à infestação pelos ácaros.

TERMOS DE INDEXAÇÃO: Demodex bovis, demodicose, bovinos, patologia, Brasil.

\section{INTRODUÇÃO}

Demodex bovis, parasita de folículos pilosos e glândulas sebáceas, está reconhecidamente associada a lesões na pele dos bovinos em várias partes do mundo. A demodicose bovina pode se apresentar de duas formas: a forma subclínica crônica com um número reduzido de pequenos nódulos e a generalizada com dezenas e/ou centenas de nódulos (Matthes 1994). A primeira é muito mais comum que a forma generalizada. A importância da forma subclínica crônica está relacionada aos danos causados 
na pele e conseqüente prejuízos na industrialização e comercialização do couro, enquanto a forma generalizada pode ser fatal (Mbuthia et al. 1994).

Do ponto de vista patológico, a progressiva multiplicação dos ácaros originam dilatações sacciformes e alargamento dos folículos pilosos e das glândulas sebáceas, formando nódulos que podem variar de alguns milímetros a centímetros de diâmetro. Os nódulos contém material caseoso de coloração brancoamarelada, onde são encontrados milhares de ácaros em todos os estágios do ciclo biológico (ovo, larva, protoninfa, deutoninfa e adulto). A fusão dos nódulos pode originar lesões do tipo granulomatosas e/ou exantemas crostosos (Bwangamoi 1970, Hutyra et al. 1973, Chakrabarti \& Pradhan 1985). Pode ocorrer ainda, invasão secundária de bactérias e/ou fungos que agravam o quadro clínico (Baker \& Fisher 1966, Bwangamoi 1970, Hutyra et al. 1973, Chakrabarti \& Pradhan 1985).

A prevalência da demodicose bovina é variável de região para região. Alguns artigos selecionados da literatura pertinente,servem de exemplo: Esuruoso (1977) reportou prevalência de 8,39 a $32,17 \%$ no Sul da Nigéria, Fisher (1974) de 82 a 93\% nos Estados Unidos da América, Murray et al.(1976) de 9 a 94\% na Austrália e Hoffman \& Hiepe (1987) de 6,5 a 52,5\% na Alemanha.

Vários fatores tais como nutrição deficiente (Griffiths 1945), estresse fisiológico causado pela gestação e lactação (Baker \& Fisher 1966, Hutyra et al. 1973), presença de outras infestações/ infecções, diferenças raciais (Nooruddin \& Rahman 1985) podem estar associados com surtos de demodicose bovina

Com relação a idade dos animais, Chakrabart \& Pradhan (1985) relataram que os animais com faixa etária entre 36 e 47 meses, apresentaram infestações de $18 \%$ enquanto os animais com idade superior a 72 meses, somente $10 \%$ estavam positivos dentre os 472 bovinos examinados. Nooruddin \& Rahman (1985), verificaram que os bovinos com idade entre 6 e 12 meses apresentaram prevalência de $7,71 \%$, entre 1 e 2 anos de idade $4,79 \%$ e os animais adultos com mais de 2 anos 0 a 2,74\%. E, em relação ao sexo, as fêmeas estavam mais parasitadas do que os machos (Chakrabart 1984, Chakrabart \& Pradhan 1985).

A literatura brasileira registra apenas citações de casos da forma subclínica desta parasitose em bovinos nos Estados de Pernambuco, São Paulo, Mato Grosso, Minas Gerais e Rio de Janeiro (Torres 1938, Rocha \& Pardi 1954, Freitas et al.1958).

Neste artigo, os autores registram pela primeira vez a ocorrência da forma clínica generalizada da demodicose bovina no Brasil.

\section{MATERIAL E MÉTODOS}

O rebanho da raça Sindhi de aptidão leiteira, pertencente à Universidade Federal da Paraíba, Campus VII, mantido na microrregião do Semi-árido do Estado da Paraíba, apresentou lesões cutâneas caracterizadas por manchas claras, escarificações e nódulos subcutâneos disseminadas no corpo dos animais. Por meio de exames clínicos, parasitológicos e histopatológicos, diagnosticou-se a demodicose bovina. Foram realizadas quatro coletas, no período entre dezembro de 1989 e janeiro de 1992, conforme Quadro 1.

O material para o exame parasitológico constitui-se de raspado profundo da pele nas áreas lesionadas (manchas claras de 1-3 cm de diâmetro e escarificações) utilizado lâmina de bisturi no.24, já que nesta fase não é possível pressionar a pele lesionada entre os dedos, como se faz com os nódulos ou material caseoso obtido através da incisão de nódulos medindo $1-3 \mathrm{~cm}$ de diâmetro com lâmina de bisturi no.24. Em ambas situações, o material obtido foi acondicionando em álcool etílico a $70 \%$.

Para o exame histopatológico, foram retirados dois fragmentos de cada nódulo, de acordo com o tamanho, $(1,0 ; 1,5 ; 2,0 ; 2,5$ e 3,0 cm), após serem medidos com paquímetro. Este material foi conservado em formol a $10 \%$. Para os diagnósticos parasitológico e histopatológico, o material coletado foi devidamente processado no laboratório utilizando-se técnicas padronizadas em Acarologia e Patologia, respectivamente.

\section{RESULTADOS E DISCUSSÃO}

Diagnosticou-se a presença de nódulos subcutâneos $(1-3 \mathrm{~cm}$ de diâmetro), os quais apresentavam-se endurecidos quando pequenos ( $1 \mathrm{~cm}$ de diâmetro) $\mathrm{e} \mathrm{bem} \mathrm{aderentes} \mathrm{à} \mathrm{pele.} \mathrm{À} \mathrm{medida} \mathrm{que}$ aumentavam de tamanho (2-3 cm de diâmetro), pareciam soltos no tecido subcutâneo. Em algumas áreas os nódulos fusionavamse formando extensas lesões semelhantes aos exantemas crostosos, com formação de tecido granulomatoso, crostas e esfoliação da pele (Fig.1). Além dos nódulos, foram diagnosticadas manchas esbranquiçadas circunscritas $(1-3 \mathrm{~cm}$ de diâmetro) dispersas em todo o corpo do animal, pêlos eriçados, alopecia, engrossamento da pele e edemaciação.

Em relação a distribuição dos nódulos pelo corpo dos hospedeiros, a região anterior compreendendo a cabeça, pescoço, barbela e paleta foi onde se diagnosticou a maioria dos nódulos, tanto nos animais mais velhos ( $>2$ anos) quanto nos mais jovens ( $<2$ anos). No primeiro grupo ( $>2$ anos), a distribuição dos nódulos em 12 animais examinados foi a seguinte: região anterior $(6 / 12=50 \%)$, região posterior $(4 / 12=33,3 \%)$ e flancos $(2 / 12$ $=16,7 \%$ ). No segundo grupo a distribuição foi a seguinte: região anterior $(7 / 15=46,7 \%)$, região posterior e flancos $(4 / 15=$

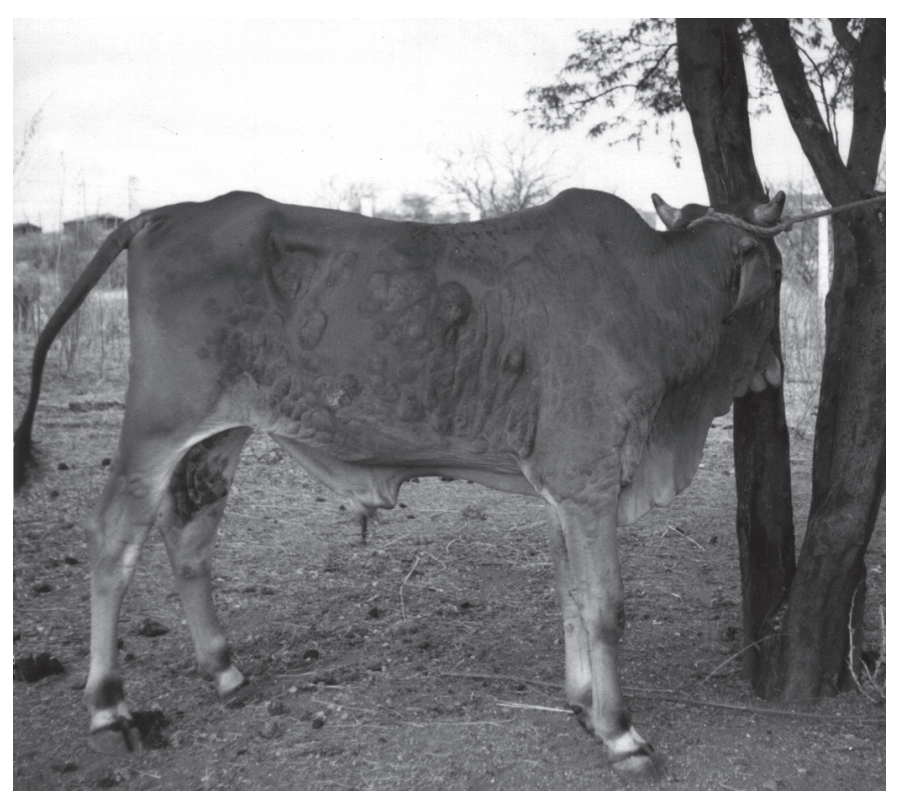

Fig. 1. Lesões nodulares na pele de um bovino causadas pela infestação por Demodex bovis. 
Quadro 1. Distribuição por faixa etária e período do ano dos bovinos com demodicose

\begin{tabular}{|c|c|c|c|}
\hline Idade & $\begin{array}{l}\text { Número de bovinos } \\
\text { examinados }\end{array}$ & $\begin{array}{l}\text { Animais } \\
\text { positivos }\end{array}$ & Prevalência (\%) \\
\hline $\begin{array}{l}\text { Em } 05 \text { e } 06 \text { c } \\
>2 \text { anos } 52 \\
<2 \text { anos } 45\end{array}$ & $\begin{array}{c}0 \\
22\end{array}$ & $\begin{array}{c}0 \\
48,9\end{array}$ & \\
\hline $\begin{array}{l}\text { Em } 20 \text { e } 21 \text { j } \\
>2 \operatorname{anos} 57 \\
<2 \text { anos } 54\end{array}$ & $\begin{array}{c}0 \\
11\end{array}$ & $\begin{array}{c}0 \\
20,4\end{array}$ & \\
\hline $\begin{array}{l}\text { Em } 15 \text { e } 16 n \\
>2 \text { anos } 61 \\
<2 \text { anos } 57\end{array}$ & 991 & $\begin{array}{l}14,8 \\
15,8\end{array}$ & \\
\hline $\begin{array}{l}\text { Em } 15 \text { e } 16 j \\
>2 \text { anos } 91 \\
<2 \text { anos } 49\end{array}$ & $\begin{array}{l}12 \\
26\end{array}$ & $\begin{array}{l}13,2 \\
53,1\end{array}$ & \\
\hline
\end{tabular}

26,7\%), cada uma. A distribuição dos nódulos está de acordo com dados publicados para outras raças bovinas, em várias partes do mundo (Mathes 1994).

A prevalência da demodicose bovina durante os 4 anos de observação está expressa no Quadro 1 . O primeiro caso da parasitose foi registrado em dezembro de 1989 , onde um animal dentre os enfermos, apresentou um quadro clínico avançado, traduzido por formação de nódulos subcutâneos que se fusionaram, formando extensas áreas de exantemas crostosos, com engrossamento e esfoliação da pele, nas regiões anteriores e posteriores do corpo do animal. $\mathrm{O}$ animal foi sacrificado devido ao seu estado geral. Este quadro clínico é compatível com as observações de Mbuthia et al. (1994) para uma vaca da raça Holandesa Preta e Branca.

Analisando-se ainda o Quadro 1, observa-se que a prevalência da demodicose nos bovinos com idade acima de 2 anos, foi diagnosticada somente à partir do terceiro ano de observação. No terceiro ano as prevalências foram semelhantes nas duas faixas etárias, enquanto que no quarto ano, a prevalência nos animais com menos de 2 anos foi cerca de quatro vezes mais alta do que a observada nos animais com idade acima de 2 anos. É importante ressaltar que em julho de 1990 , apesar de ser considerado um mês seco, tradicionalmente, ocorreram chuvas escassas e não definidas, que permitiram o crescimento de pasto nativo. A utilização destas pastagens com suplementação alimentar (forrageiras, leguminosas, silagem) possibilitou a recuperação de alguns animais, resultando na regressão dos nódulos subcutâneos e diminuição do número dos mesmos, como também das manchas esbranquiçadas.Little \& Princeton (1932) e Griffiths (1945) já haviam observado também que vacas em período de lactação e animais sem alimentação adequada apresentaram exacerbação clínica da demodicose. Verificaram também que a separação do bezerro e alimentação adequada resultou na regressão gradativa dos nódulos.

No período de março de 1991 a janeiro de 1992, com o incremento da estiagem, embora o período fosse de chuva, houve um aumento gradativo da parasitose nos animais com idade acima de 2 anos e uma nova exacerbação dos sinais clínicos, principalmente nos animais jovens, onde as lesões foram multifocais e difusas com características de exantemas crostosos e esfoliação da pele, semelhantes ao primeiro animal que foi sacrificado. As observações aqui registradas sugerem que o estresse nutricional foi responsável pela exacerbação da demodicose bovina. $O$ surgimento das lesões nos animais acima de 2 anos de idade mais tardiamente do que naqueles com menos de 2 anos (Quadro 1) sugere que animais mais velhos são mais resistentes, nas condições aqui relatadas.

Os resultados descritos neste artigo discordam das afirmações de Podestá et al. (1980), os quais relataram que os animais jovens do México e da Costa Rica não são acometidos pela parasitose.

A patologia aqui relatada foi observada tanto nos machos como nas fêmeas.

Embora a distribuição da parasitose seja cosmopolita, pouco se conhece sobre sua epidemiologia no Território Nacional. Os dados coligidos dessas observações, somados aos registrados na literatura brasileira, permitem-nos inferir que a parasitose ocorre em todo o Território Nacional. Tanto as raças zebuínas como as européias podem ser parasitadas, assim como o sexo e

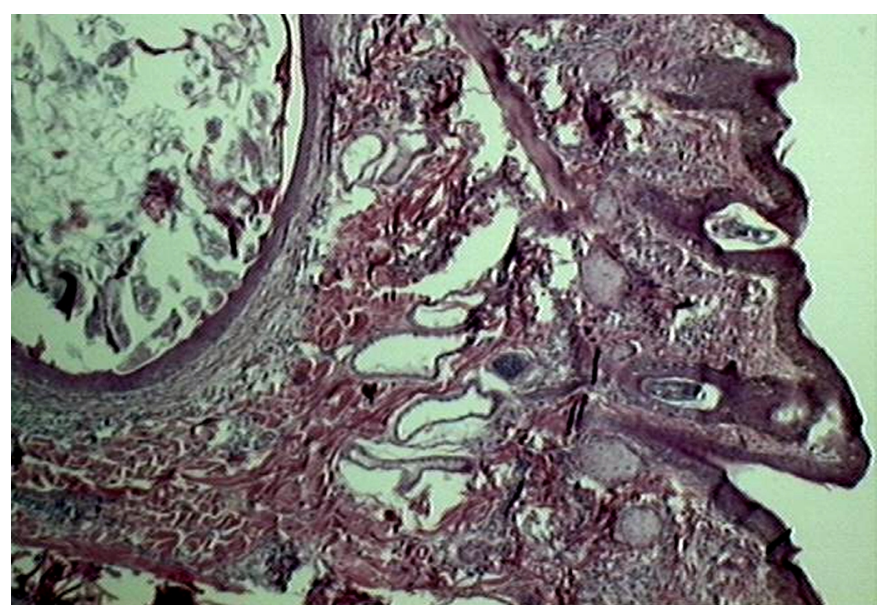

Fig. 2. Foliculite demodécica em bovino, com acantose, dilatação linfática, de glândulas sudoríparas e de folículo piloso na derme profunda com o lúmen repleto de exemplares do ácaro. HE, obj.5.

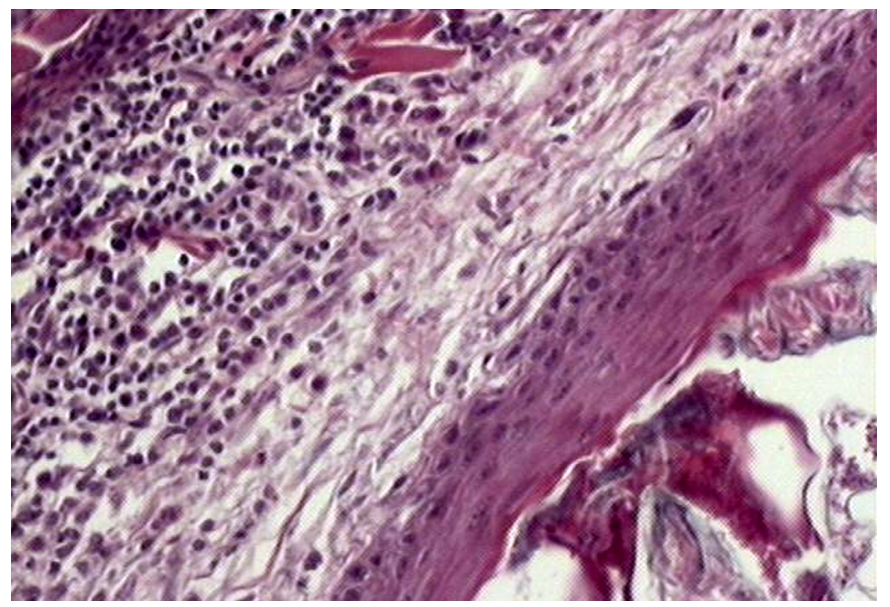

Fig.3. Foliculite demodécica em bovino, observando-se infiltrado linfoplasmocitário perifolicular. HE, obj.20. 
a idade não são fatores limitantes. A parasitose é raramente fatal, apenas animais debilitados e em determinadas condições estressantes morrem.

Nos raspados e no material caseoso obtido do interior do nódulo, detectou-se a presença dos ácaros, os quais foram isolados e identificados como Demodex bovis.

Nos cortes histológicos corados pela hematoxilina-eosina e tricrômico de Mallory diagnosticaram-se acantose com hiperqueratose, adenite sebácea crônica, necrose muscular subcutânea, degeneração focal de células da camada basal da epiderme, presença de grande número de ácaros de localização intraluminal nos terços médio e inferior de folículos pilosos dilatados e perifoliculite crônica, caracterizada por infiltrado celular predominantemente linfoplasmocitário, contendo ainda macrófagos e neutrófilos, $\mathrm{O}$ infiltrado linfoplasmocitário envolvia bulbos pilosos, induzindo os folículos a entrar em fase de repouso do ciclo piloso, acarretando a queda de pêlos (alopecia). As Figuras 2 e 3 exemplificam algumas destas lesões. Em linhas gerais, o quadro patológico descrito neste artigo é muito semelhante aqueles descritos na literatura (Nemeséri \& Széky 1961, Mbuthia et al. 1994).

As alterações patológicas provocadas na pele são importantes por ocasião da industrialização das mesmas, resultando em um couro de qualidade inferior acarretando sérios prejuízos econômicos (Fisher 1974).

\section{REFERÊNCIAS}

Baker D.W. \& Fisher W.F. 1966. Demodectic parasites in livestock. 7th Annu. Meet.US Livestock Sanit. Assoc., Local?, p.409-416.

Bwangamoi O. 1969. A survey of skin diseases of domesticated animals and defects with downgrade hides and skins in East Africa. Bull. Epizoot. Dis. Afr. 17:197-203.

Chakrabarti A. 1984. Some epidemiological features of bovine demodicosis. Indian J. Vet. Med. 4:80-83.
Chakrabarti A. \& Pardhan N.R. 1985. Demodicosis in livestock in West Bengal (India). Int. J. Zoon. 12:283-290

Esuruoso G.O. 1977. Bovine demodicosis in Southern Nigeria. Bull. Anim. Health Prod. Africa 25:65-72.

Fisher W.F. 1974. Incidence of demodicosis in commercially pickled steerhides. J. Am. Leather Assoc. 69:5-10.

Freitas M.G., Costa H.M.A. \& Leonhardt H.G. 1958. Sarna demodécica dos bovinos em Minas Gerais. Arq. Esc. Sup. Vet., Belo Horizonte, 11:375377.

Griffiths J.A. 1945. Demodectic mange of domestic animals in Nyassaland. J. Comp. Path. Therap. 28:61-66.

Hoffman G. \& Hiepe T. 1987. The present status of bovine demodicosis in the GDR. Monatsh. Veterinärmed. 42:704-708.

Hutyra F., Marek J. \& Manninger R. 1973. Patologia y Terapêutica Especiales de los Animales Domésticos. Vol 2. Labor, Barcelona. 1033p.

Little B.R. \& Princeton N.J. 1932. Demodectic (follicular) mange in cattle. J. Am. Vet. Med. Assoc. 80:922-926

Matthes H.F. 1994. Investigations of pathogenesis of cattle demodicosis: sites of predilection, habitat and dynamics of demodectic nodules. Vet. Parasitol. 53:283-291.

Mbuthia P.G., Kariuk D.I. \& Mulei, C.M. 1994. Generalized demodicosis in an Friesian heifer from a zero-grazing unit. Vet. Parasitol. 51:337-343.

Murray M.D., Nutting W.B. \& Hewetson R.W. 1976. Demodectic mange of cattle. Aust. Vet. J. 52:49.

Nemeséri L. \& Széky A. 1961. Demodicosis in cattle. Acta Vet. Acad. Sci. Hung. 11:209-221.

Noorudin M. \& Rahman S.M. 1985. A note on bovine demodicosis in Bangladesh. India J. Vet. Med. 5:139-140.

Podestá M., Quirós J., Cordeiro L. \& Alvarado, R. 1980. Diagnóstico clínico de la demodicosis em el ganado bovino. Cienc. Vet., México, 2: 169176.

Rocha U.F. \& Pardi M.C. 1954. Sarna em bovinos do Estado de São Paulo causada por Demodex bovis (Stiles, 1892) sic. Acari: Demodicidae. Revta. Fac. Med. Vet., São Paulo, 5:27-34.

Torres S. 1938. Sarna demodética em bovinos. Bolm Secr. Agric. Ind. Com. Pernambuco 3:123-124. 